\title{
Disorderly Reasoning in Information Design
}

Forthcoming paper:

Disorderly Reasoning in Information Design

By Peter A. Hall

In Journal of the American Society for Information Science and Technology, Perspectives, Special Issue:

Design

Wiley Periodicals, Inc. (Spring 2009)

Biographical Sketch:

Peter Hall is a design critic, and senior lecturer in design at the University of Texas at Austin. Recent publications include Else/Where: Mapping - New Cartographies of Networks and Territories (co-edited with Janet Abrams, University of Minnesota Design Insititute, 2006) and "Diagrams and their Future in Urban Design" in Garcia,M. (Ed.), Diagrams of Architecture (Wiley 2009)

\footnotetext{
Abstract

The importance of information visualization as a means of transforming data into visual, understandable form is now embraced across university campuses and research institutes worldwide. Yet the role of designers in this field of activity is often overlooked by the dominant scientific and technological interests in data visualization, and a corporate culture reliant on off-the-shelf visualization tools. This article is an attempt to describe the value of design thinking in information visualization with reference to Horst Rittel's definition of “disorderly reasoning,” and to frame design as a critical act of translating between scientific, technical and aesthetic interests.

In a discussion of the aesthetics of information visualization, Warren Sack addresses a common misconception of artistic data visualization as "an exercise in beautiful image-making to render data “friendly"”(Sack, 2009). This characterization is, according to Sack, "unsatisfactory for most artists and designers concerned with information visualization. It is tantamount to an understanding that the artistic work is only an attempt to "pretty things up,” i.e., to make computer images easy to understand.” Sack’s work is helpful in its efforts to define a new aesthetics of information and the network, and its characterization of a prevailing view of aesthetics as superficial. The work of many artists using data visualization is more user-unfriendly in its critical modes of challenging and calling attention to network conventions. But to dismiss user friendliness as "prettying things up” is to throw out the baby with the bath water.
} 
In a concurrent essay, Sacks elaborates on the philosophical problems of terms like "user friendly" -which depends on culturally specific notions of common sense in a multi-cultural medium like the Internet(Sack, 2009). But as mediators between art and science, designers depend on loose concepts like user-friendliness in order to proceed, based on somewhat unscientific hunches about the given audience for a specific visualization. The postmodern philosopher Jean Lyotard's characterization of the profession is surprisingly apt:

"Graphic artists cannot make a living without making hypotheses about the public. This is not a civilization nor even a culture in the anthropological sense. This is the combination, endlessly unmade and remade, of temporary sensibilities” (Lyotard, 1999).

There is no widely-accepted fixed definition of design, a point that can be considered characteristic of and beneficial to a relatively new field of learning (Buchanan, 2001). However, a useful corrective to prevailing notions that the designer comes to "pretty up" a project after all the important technical, economic, social and ethical decisions have been made, can be found in mathematician and design theorist Horst Rittel's notion of the "wicked problem”. According to Rittel’s essay, “The Reasoning of Designers” (1998), designing is plan-making that takes place in a social context and is characterized by a subjective process of argumentation. Designers tackle the kinds of problems that cannot be solved by traditional and formulaic processes, but by a "disorderly reasoning”:

"A design problem keeps changing while it is treated, because the understanding of what ought to be accomplished, and how it might be accomplished is continually shifting. Learning what the problem is IS the problem.”

Significant to Rittel's concept of design is that it is fundamentally humanist, assuming that designers share an ambition to "imagine a desirable state of the world". Applied to information visualization, Rittel's account of design allows us to accept both the idea that designers might make data "friendly" but they also would recognize when conventions of data-friendliness may not provide an adequate criterion for identifying a design solution. In Rittel's account a designer is (or should be) in a position to ask the critical question, “Am I dealing with the appropriate problem, or is this problem only a symptom of some other, higher level problem which I should attack instead?” (Rittel, 1998).

\section{Interdisciplinarity and Network Mapping}

Clearly, once visualization designers begin to talk about "higher level problems", they move out of what might be considered the traditional jurisdiction of, say, graphic or user interface designers, into fields that require other sorts of expertise. Yet to do otherwise can seem increasingly myopic given the increasing complexity and interdependence of problems faced by designers. Indeed, the flurry of recent interest in 
“design thinking”, including considerable investment in new institutions such as Stanford Institute of Design's D-School, can be seen as the application of Rittel's ideas. The D-School's promotional material describes its mission with an implicit nod to Rittel: "We tackle difficult messy problems that demand interdisciplinary solutions.”

Collaborative, interdisciplinary work is crucial to higher level thinking: Rittel describes design reasoning as characterized by an "epistemic freedom," meaning that it is not governed by logical or epistemological rules, and grants that "planners, engineers, architects, corporate managers, legislators, educators are (sometimes) designers" (Rittel, 1998). Significantly, one archetypal work of information visualization often lionized by graphic designers is the work of an engineering draughtsman.

In 1931, Harry Beck sketched a diagram of the rapidly expanding London Underground system that abstracted the underground train system from the geography in which it was embedded, and schematized it, based on the model of an electrical circuit. Despite initial rejection by London Transport authorities, the map was tested, proved enormously popular and has since become the model for metropolitan transport maps around the world, as well as a navigational mnemonic, a concise representation of London's sprawling metropolis. Beck was not commissioned to solve a visualization problem; he defined it, not coincidentally, during a time when London Transport had laid him off. His disorderly reasoning was in recognizing the need to provide a clear navigational diagram for the growing system (which was operating under capacity at the time) was thwarted by cartographic traditions, which in prioritizing the actual location and distance between stations produced meandering, disorienting maps. (Garland, 1994).

As a means of representing information, Beck's diagram was prescient in that it anticipated the decreasing importance of geographical proximity in the circuits of production, foretelling the rise of today's network society. Topographic network maps have since become a standard means of representing networks of all kinds, from social networks to organizational charts to fields of knowledge to the traffic between IP addresses. The dominance of this kind of visualization comes at a cost, however, since it imposes a metaphor on subjects that behave quite differently from electrical circuits.

As is well known by Londoners, Beck's diagram is an unreliable map of the actual city, depicting long distances and complex routes between stations that, above ground, are within a few hundred feet of each other (Wimbledon and South Wimbledon, or Queensway and Bayswater are classic examples). Designer and writer Paul Elliman notes that its dominance as a form reveals the degree to which the language of visual representation can "shape a mental image of a city, inscribing inaccurate or vague senses of distance, scale and direction, and giving prominence to certain places simply by virtue of their being selected for the map.” (Elliman, 2006). Elliman observes the prevalence of city-as-machine metaphors at the time Beck created the diagram, an emerging urban vocabulary of reticular terms and the subsequent characterization 
of passengers as message bearing systems. Henri Lefebvre's skepticism of the modern city as functionalist paradigm identifies a political subtext of the network map:

"To claim that the city is defined as a network of circulation and communication, as a center of information and decision making, is an absolute ideology.” (Lefebvre, 1996)

The idea that every cartographic map has an agenda has been well established, by mapmakers like Denis Wood (Wood, 1992), and scholars like JB Harley, who put it succinctly: "maps redescribe the world...in terms of relations of power and of cultural practices, preferences and priorities” (Harley, 2001). Yet network maps continue to assume a veil of neutrality. Elliman re-reads Beck's map by casting the simple design problem of depicting a transportation network as a thicker problem. A similar critical analysis might be applied to the widespread use of topological network maps in representing social networks.

In a paper titled "The Social Life of Routers," for example, management consultant and network analyst Valdis Krebs draws analogies between computer and human networks in order to identify key personnel or subject matter experts in a corporation's network of knowledge assets (Krebs, 2000). In one instance, Krebs uses a simple hypothetical social network diagram to debunk the common wisdom that "more connections, the better" by showing that certain, less connected people are sometimes located in key positions between important constituencies--“similar to that of a border router”. Implicit in the design of the topological network visualization, however, are several assumptions about the rate of transfer of knowledge around a network and the working atmosphere. Obviously a work environment in which people share knowledge freely in pursuit of a shared goal will lessen the impact of a key figure (the "border router") departing the network, compared with an environment in which long-entrenched employees harbor their knowledge as a form of power. The missing information from Krebs’ map may ultimately provide the key to the functioning of the network, to the extent that a map of the mood of the network may be more useful.

Concurrent with Krebs' social network mapping researchers in the Sociable Media Group at MIT, led by Judith Donath, began exploring means of visualizing anger on discussion groups. The experiments formed part of a project exploring alternative visualizations to browsers, which the MIT team felt are ill-equipped to reveal the "rich social features" of a newsgroup (Donath, 2002). In asking questions of today's social networking websites, Donath notes the primitive nature of their structures: "Their notion of ties is uniform - they make little distinction between the ties connecting close friends and vague acquaintances. Nor is there any recognition of the faceted nature of social life. People associate with different groups, consonant with different facets of their lives: they may not wish to make those connections known to each other.” The critique applies equally to visualizations of social networks, which also tend to make links between nodes 
uniform, and ignore the fact that some nodes (people) prefer to keen links (connections) hidden. Donath noted in a discussion on the topic:

“This is a big problem in the mapping of any social data - such as conversations - whereby fixing something onto an image, we give it a solidity it does not merit” (Donath, 2006)

Networks are, by definition, continually in flux, and links between nodes can vary in strength; efforts have been made to distance nodes spatially according to the strength of ties, (“loaded spring” maps) and to reflect kinds of ties with the thickness of connecting lines, but it is inherent to the design of a network diagram that connections appear certain and fixed. (This connotation gives rise to workarounds like dotted lines signifying subway train lines under construction and pencil lines signifying speculation, as in the work of artist Mark Lombardi). Media writer JJ King contends that information cartography is not yet capable of capturing the "tacit, affective and transversal qualities of the knowledge economy” (King, 2006).

\section{The User Interface: Visualization Monopolized?}

To develop a description of the designer as a bridge between the critical considerations that underlie art practice with the practical problem-solving, or plan-making, aspects of design as it interfaces with science and technology, it is helpful to examine to two designer-driven critiques of standard, everyday visualization tools, namely PowerPoint and the Windows/Mac OS desktop metaphor. Although it might be contended that both examples are of graphic user interfaces rather than information visualizations, I side with current thinking that casts information visualization and user interface design as subsets of the larger field of human-computer interaction. Both are interactive tools that permit a "conversation with data” that may or may not require dense information. (Shirkey, 2002). In order to make information on computers accessible, it must be visualized.

Edward Tufte's polemic against a ubiquitous software tool, “The Cognitive Style of PowerPoint: Pitching Out Corrupts Within” (Tufte, 2006) finds that as a means of visualizing information, Microsoft's slide presentation software, which turns out "billions and billions of presentation slides a year”, suffers a litany of deficiencies. Tufte's complaints are extensive, but chief among them is that its user interface encourages the abbreviation of evidence and an aggressive, pushy style of delivery mimicking the software house itself:

“A big bureaucracy engaged in computer programming (deep hierarchical structures, relentlessly sequential, nested, one-short-line-at-a-time) and in marketing (advocacy not analysis, more style than substance, misdirection, slogan thinking, fast pace, branding, exaggerated claims, marketplace ethics.)" 
The smoking gun in Tufte's prosecution is a set of PowerPoint reports prepared for NASA by engineers at the Boeing Corporation to address the damage impact of foam debris falling during from the space shuttle Columbia in January 2003. The report was compiled during the shuttle’s two-week orbit after video capture recorded the foam insulation breaking off the fuel tank and hitting a wing, causing (unbeknownst to the engineers) a hole that would ultimately cause the shuttle to burn up on re-entry into the Earth's atmosphere, killing seven astronauts on board. Tufte contends that PowerPoint's “hyper-rational” hierarchy abbreviated and then de-prioritized or deleted important data that would have indicated to NASA that Boeing's test data was irrelevant rather than reassuring, as NASA management officials assumed from the slides. Both the 2003 Columbia Accident Investigation Board report and the Final Report of the Return to Flight Task Group (2005) concurred that the endemic use of Powerpoint briefing slides instead of technical papers highlighted a problem with technical communication methods at NASA.

Having expertly identified the way in which ideological and cultural factors influenced the development of software, Tufte diplomatically proposes as a solution the use of Microsoft's word-processing software in place of PowerPoint for technical papers. More convincing is the manner in which his detailed attack on a presentation tool illustrates the argument that no user interface is neutral. To trace the development of a dominant piece of software is to encounter a sequence of problem definitions that simplify and displace interests and goals in the course toward creating an "obligatory passage point,” to use the sociological term of Michel Callon (1986). John Shiga tracks the development of Apple's iPod in this fashion, through a sequence of problem definitions from the International Standards Organization's first identification of digital audio compression as a target goal in 1988, to engineers' adoption of a psychoacoustic model of hearing in 1995, to the development of MP3 and AAC and then proprietary digital rights management (DRM) formats by Apple (Shiga, 2006). This way, Apple’s iTunes became “an online music service and tool for selling and promoting Apple’s other products, rather than an application for playing music acquired elsewhere. MP3 players become extensions of an online music store rather than devices for playing music downloaded from file-sharing networks or ripped from CDs.” (Shiga, 2006, p. 43) At a certain point, the dominant technology comes to seem natural, even neutral.

The same can be said of the Internet, which has infiltrated everyday life to the point that the connotations of a technology developed in an atmosphere of Cold War paranoia and surveillance are given barely a thought as we voluntarily upload increasing amounts of personal data online. Alex Galloway argues convincingly that the popular conception of societal control disappearing amid decentralized communication technologies is fundamentally misconceived. With the distributed network comes a more insidious kind of control that operates in the protocol through which we move information about: "Protocol is to control societies as the panopticon is to disciplinary societies... While protocol may be more democratic than the panopticon in that it strives to eliminate hierarchy, it is still very much structured around command and control” (Galloway, 2004). He allows, nevertheless, for a Deleuzian explanation of the burgeoning of net 
art, adding that protocol, because of its command and control structure, has "spawned counter-protological forces.”

Galloway notes a number of recent examples of "net art" that draws attention to the fabric and structure of the internet itself, citing, among many examples of this renegade art form, the work of the European duo Jodi, whose work focuses on the places where computers break down, on the protocols of the internet, with their technical peculiarities, and deals "creatively with software and with the rules software follows in order to work.”

Jodi's OSS project (http://oss.jodi.org) takes over the functions and appearance of the desktop and pulldown menus, transforming the graphic user interface into various inoperable forms, fragmented and violently jumping around the screen. Following Sack we can situate such digital mischief as the genealogical descendent of 1960s conceptual art like Sol Lewitt's, which employed bureaucratic criteria as its working method, miming the operating logic of late capitalism. Sack cites Alan Turing's 1936 paper articulating the principles of contemporary computing by comparing bureaucratic techniques with the mental activities of man, arguing that "computers are an outgrowth of bureaucracy” (Sack, 2009).

The cultural specificity of the desktop metaphor, with its hierarchical file-folder system, office wastebasket and, in more recent versions of Windows and Macintosh operating systems, excess of operational buttons and functions, is a key consideration for designers as well as net artists. For the development of Sugar, the Linux-based user interface of the One Laptop Per Child (OLPC) initiative, bureaucratic metaphors were considered inappropriate for a low-priced XO Laptop aimed at children in developing countries with no prior computer experience, or familiarity with file folders. Although the makers of the operating system describe Sugar as an "extension” (OLPC, 2008) rather than a rejection of the desktop metaphor, it depends on, and implicitly embodies, a critique of its desktop forebears.

In place of the personalized desktop, overlapping windows and filing system, the designers of Sugar (Pentagram Design and Red Hat, led by Nicholas Negroponte and Walter Bender) developed a new fullscreen environment that depicts the operator as a color-coded stick figure in the center of the screen, surrounded by activity icons and other children connected to the laptop’s ad hoc "mesh" network (a "selfhealing”, modular network in which connections can be reconfigured around broken paths by hopping from node to node). A zoom-based interface allows children to switch between three views: a neighborhood view shows all connected users and their activities; a group view shows children's shared activities; and a home view allows them to start new ones, or arrange their favorites. Activity icons assume the same color code as the child once started. 
Critics of the Sugar interface take issue with its developers' decision not to test the interface in advance, and question the value of learning a brand new operating system when "real" computers are generally Windows or Macintosh-based. ${ }^{i}$ The OLPC team contends that computer literacy (with conventional software applications) is a by-product of computer fluency, which would be gained through the use of the XO laptop for learning: "Children—especially young children—need the opportunity to learn far more than Word, Excel, and Powerpoint” (OLPC, 2008). The rationale for not user testing Sugar seems to be based on a degree of entrepreneurial hubris: the theoretical underpinnings of the project lie not in Taylorist or bureaucratic ideas but Constructionism, a theory of learning defined by Seymour Papert, an advisor to OLPC. Drawing from the writings of Jean Piaget, Papert defines Constructionism as a combination of the Constructivist theory of learning as a reconstruction rather than transmission of knowledge, with the idea that learning is most effective when it is experienced as part of constructing a meaningful product (Papert, 1989).

The idea that some innovations require a kind of hubris, or single-mindedness that disregards user-testing protocol or other product development conventions is, of course, familiar territory to designers. Accounts of designers who persuaded their clients (or themselves) to buck convention and forge ahead with their (sometimes well-grounded) ideas are legion, and can be found in accounts of genre-defining products as the Herman Miller Aeron Chair, the Palm Pilot and the downloadable ringtone ${ }^{\mathrm{ii}}$. While such accounts should be treated with skepticism, since they often veil the presence of other actors or influences in a product's development, user testing is clearly not prioritized in the process. Rittel's description of disorderly reasoning picks up on this hubris:

" Designers think more or less coherently; they figure, they guess, they have sudden ideas "out of the blue”, they imagine, speculate, dream, let their fantasy wheel freely, scrutinize, reckon, they 'syllogize’” (Rittel, 1988).

Rittel's philosophy is not of the Fountainhead variety. He notes wryly at the end of "The Reasoning of Designers":

"Fortunately for all of us, most designers don’t succeed in shaping the world their way. Design takes place in a social context."

The possibility remains that OLPC is a product of an overly rigid and top-down development and distribution structure destined to fail amid the intensely wicked problems of equipping children in developing countries with computers, and then mentoring them. Rittel's point is not to side with the visionary designer and his "out of the blue" thinking at all costs, but to allow for a disorderly approach to the complexities of design problems, and emphasize that design is never objective; it takes sides and as such is political. 
Sugar is a particularly interesting contribution to information visualization because it explores a different paradigm to a "good enough" operating system (Windows/Mac) in an advanced stage of feature-itis. Its development seems disorderly because it both embraces and avoids standard developer procedures. One particular detail of the XO user interface echoes an organizational system used in Googledocs, which might also be viewed as a new paradigm for a graphic user interface based on collaborative work, rather than solitary desktop-based processing. In Sugar, a journal automatically records the child's interactions, projects and collected media, and organizes it, as does Googledocs, according to time, so that most recent projects are at the top of the list. Since the storage capacity of the laptop's flash drive is relatively small, an automated back-up system archives on a server projects that have not been used for a specified period of time. Items can be deleted, but the activity is not prioritized (throwing things into the trash is also a culturally specific occupation.) Google’s Gmail uses a similar principle, offering an ever-expanding storage capacity to allow account holders to avoid purging old, "heavy” emails.

\section{Visualizing Conversations}

The Web has become an extraordinarily rich field for the flourishing of visualization methods, notably in the hands of non-professionals. Relatively new methods of arraying statistical data, like the treemap, developed by Ben Shneiderman in the early 1990s for space-constrained visualization of hierarchies, have gained popularity on the Web, most famously with the Smart Money magazine map of the market (Wattenberg, 1999). Other, classic statistical diagrams such as the scatterplot, stack graph and line graph, have developed into animated and interactive forms. While the possibility for misrepresentation of data increases with the popularization of visualization, the field has gained a massive test bed of volunteer users, creating, viewing and discussing visualizations.

Recognizing this phenomenon, Martin Wattenberg and Fernanda Viégas, research scientists at the Visual Communication Lab at IBM Research group, coined the phrase "vernacular visualization" to describe the ubiquitous tag cloud, a relatively imprecise visualization tool that nevertheless has risen to prominence with the advent of the social networking and user-participation sites of the Web 2.0 era (Viegas \& Wattenberg, 1999). Wattenberg and Viegas find precursors of the tag cloud in a 1976 experiment conducted by psychologist Stanley Milgram, in which subjects were asked to name landmarks in Paris, from which a collective mental map of the city was formed, using larger font sizes to represent places mentioned more often. The method was adopted by Flickr, the image-sharing site, to represent how contributors had tagged their photographs in 2002, with point size attributed according to the popularity of tags ("wedding," "family" and "party" are currently among the most prominent). Tag clouds have since proliferated, proving a popular way of representing collective activity on the fly, and of comparing the content of texts, notably the transcripts of political speeches. As Wattenberg and Viégas point out, these unruly word maps have obvious flaws as information visualizations: point sizes are difficult to compare when they are not adjacent; long words receive undue emphasis; and alphabetical ordering means that related words (like "East" and 
"West”) are scattered. Their popularity seems to have more to do with their social function, providing an informal point of entry into a complex site. Wattenberg and Viégas conclude:

"the value users draw from such visualizations, as social signalers or as tools for collective text analysis, suggests that experts in information design might want to rethink the purpose and goals of their creations.”

In this sense the vernacular visualization has the benefit over the network map of not appearing fixed or certain in its loose aggregations of key words. No one would consider using a tag cloud to represent a subway system, but as a device for comparing documents or speeches it can shed immediate light on the key themes. In a similar pursuit of new means of bringing multi-user processes to light, Wattenberg and Viegas developed the "history flow map" in order to track amendments to Wikipedia entries by multiple users. The colorful chart does a better job of revealing the ebb and flow of activity on an entry-compared with the side by side comparison of near-identical text documents that Wikipedia offers. A close reading of a history flow map of entries on the topic of abortion shows spikes of activity and large gashes where the entire text has been removed and replaced with offensive remarks, a common type of vandalism on controversial topics.

\section{Conclusion}

Every visualization, of course, is an abstraction, selective in its choice of what to show, and the glib conclusion would be to suggest that there is an appropriate visualization for each particular body of data. This would be to ignore the point that a visualization, and its politics, begins not with the method, nor even with the gathering of data, but further back, with the identification and framing of a subject from which to derive information. Data is never neutral. A more interesting conclusion is to be gleaned from the phenomenon of collective data analysis which becomes possible only with collaborative online tools.

Every day thousands upon thousands of readers are examining visualizations with an unprecedented level of visual literacy. With the increasing availability of visualization tools, and sites like Wattenberg and Viegas's “Many Eyes”, which provides user-uploaded data sets (40,000 to date) and 14 types of visualization, the art of designing information is being democratized (Viegas, 2007). Non-programmers and amateur statisticians are developing their own understanding of how information is shaped and traded. A map, as JJ King points out, is only as useful as the analysis brought to bear upon it, and one can hope, with Wattenberg and Viegas, that a visually literate readership is providing a public testing ground for new knowledge and its representation (King, 2006).

The flipside of this scenario, of course, is that such wiki-utopianism gives way to mob rule, and the dominance of visualization defaults, as Tufte's cautionary tale of PowerPoint at NASA revealed, will drag down the critical discourse. It is up to designers of all descriptions, as mediators between art and science, to 
ensure that a "disorderly reasoning" is brought to the problem of how, why and which information should be visualized.

Buchanan, R (2001). Design Research and the New Learning. Design Issues: Volume 17, Number 4 Autumn 2001. Massachusetts: MIT Press

Callon, M. (1986). Elements of a sociology of translation: Domestication of the Scallops and the Fishermen of St Brieuc Bay. In John Law (Ed.), Power, Action and Belief: A New Sociology of Knowledge? (pp. 196-233). London, Routledge

Donath, J., Boyd, D., Lee, H., \& Ramage, D. Loom 2 (2002). Visual Complexity. MIT - Media Lab. Retrieved

March 10, 2009 from http://www.visualcomplexity.com/vc/project details.cfm?id=402\&index=402\&domain= Donath, J. (2007). Social Networks. In J. Donath, Signals, Truth and Design. MIT Press (forthcoming).

Donath, J et al. (2006). Conversations as Maps, Part 1. In J. Abrams and P. Hall (Eds.) Else/Where: Mapping

- New Cartographies of Networks and Territories (p. 78). Minneapolis: University of Minnesota Design Institute

D-School, Hasso Platner Institute of Design at Stanford. Retrieved March 10, 2009 from

http://www.stanford.edu/group/dschool/projects/community_impact.html

Elliman, P. (2006). Signal Failure. In J. Abrams and P. Hall (Eds.) Else/Where: Mapping - New

Cartographies of Networks and Territories (pp. 166-175). Minneapolis: University of Minnesota Design Institute Garland, K. (1994). Mr Beck's Underground Map: A History. London: Capital Transport Publishing. Press Galloway, A. (2004). Protocol: How Control Exists After Decentralization. (p. 13). Cambridge, Mass: MIT

Harley, JB. (2001). The New Nature of Maps: Essays in the History of Cartography. Baltimore: John Hopkins University Press

Hearst, M., and Rosner, D. (2008). Tag Clouds: Data Analysis Tool or Social Signaller? In Proceedings of HICSS 41, 2008

King, JJ. (2006). The Node Knows. In J. Abrams and P. Hall, Else/Where: Mapping - New Cartographies of Networks and Territories (p. 49). Minneapolis: University of Minnesota Design Institute

Lefebvre, H. (1996). Philosophy of the City and Planning Ideology. In E. Kofman and E. Lebas, (Eds.), Writings on Cities. (p. 98). Cambridge, Mass: Blackwell Publishing.

Lyotard, J. (1999). Paradox on the Graphic Artist. In Postmodern Fables. (pp. 33-47). Minneapolis:

University of Minnesota Press

One Laptop Per Child. (November 2008) Overview. Retrieved March 10, 2009 from

http://www.laptop.org/en/laptop/software/index.shtml

Papert, S. (1989). NSF Proposal. Retrieved March 10, 2009 from

http://nsf.gov/awardsearch/showAward.do?AwardNumber=8751190 April 10, 1989

Rittel, H. (1988). The Reasoning of Designers. In Arbeitspapier A-88-4. Stuttgart: Institut für Grundlagen der Planung, Universität Stuttgart

Sack, W. (2009). Aesthetics of Information Visualization (forthcoming) in C. Paul, V. Vesna, and M. Lovejoy (Eds.), Context Providers. Retrieved March 10, 2009 from

http://hybrid.ucsc.edu/SocialComputingLab/publications.htm

Sack, W. (2009). Network Aesthetics (forthcoming). In V. Vesna (Ed), Database Aesthetics. Minneapolis:

University of Minnesota Press. Retrieved March 10, 2009 from

http://hybrid.ucsc.edu/SocialComputingLab/publications.htm

Shirkey, C. (2002). Information Visualization: Graphical Tools for Thinking about Data (2002). Release 1.0,

Vol 20, No.8, September 2002. New York: Edventure Holdings

Schmidt, C.S. (2008). Email correspondence, November 10, 2008

Shiga, J. (2006).Translations: Artifacts from an Actor-Network Perspective. In Artifact, 1:1. (pp. 40-55).

London: Routledge, April 2006.

Tufte, E. (2006). The Cognitive Style of PowerPoint: Pitching Out Corrupts Within. In Beautiful Evidence

(pp. 157-185). Cheshire: Graphics Press

Viégas, F., Wattenberg, M., Van Ham, F., Kriss, J., \& McKeon, M. (2007). Many Eyes: A Site for Visualization at Internet Scale. IEEE Infovis. October 2007.

Viégas, F \& Wattenberg, M. (2008). Tag Clouds and the Case for Vernacular Visualization. In ACM

Interactions, XV.4 - July/August, 2008

Wattenberg, M. (1999). Visualizing the Stock Market. Association for Computing Machinery Computer

Human Interaction. May 1999. Smart Money Map of the Market. Retrieved March 10, 2009 from

http://www.smartmoney.com/map-of-the-market/

Wood, D. (1992). The Power of Maps. New York: Guilford Press 
i See, for example, http://www.ixda.org/discuss.php?post=23928 and

http://www.fonly.typepad.com/fonlyblog/2005/11/problems_with_t.html

ii See, for example, Gladwell, M. (2007) The Chair of Death. In Blink (p. 67). Back Bay Books 